\title{
Combining Pervasive Technologies and Cloud Computing for COPD and Comorbidities Management
}

\author{
I Chouvarda, V Kilintzis, K Haris, V Kaimakamis, E \\ Perantoni, N Maglaveras \\ Lab of Medical Informatics, Aristotle University \\ Thessaloniki, Greece
}

L Mendes, C Lúcio, C Teixeira, J Henriques, P de

Carvalho, RP Paiva

Centre for Informatics and Systems, University of Coimbra

Coimbra, Portugal

\author{
S D'Arcy \\ Clinical Research Centre, Royal college of Surgeons in \\ Ireland \\ Dublin, Ireland
}

\author{
N Philips \\ Medical Information and Network Technologies research \\ Centre, Faculty of Science, Engineering and Computing, \\ Kingston University \\ Kingston, UK
}

O Chetelat, J Wacker, M Rapin, C Meier, J-A Porchet Centre Suisse d'Electronique et de Microtechnique

Neuchâtel, Switzerland

I Frerichs

Dept. Anaesthesiology and Intensive Care Medicine University Medical Centre Schleswig-Holstein

Kiel, Germany

\author{
Andreas Raptopoulos \\ EXUS \\ Athens, Greece \\ arap@exus.co.uk
}

\begin{abstract}
Integrated care of patients with COPD and comorbidities requires the ability to regard patient status as a complex system. It can benefit from technologies that extract multiparametric information and detect changes in status along different axes. This raises the need for generation of systems that can unobtrusively monitor, compute, and combine multiorgan information. In this paper, the concept and ongoing work for such an approach is presented as regards the multiple types of data recorded, features extracted, and examples of how they are combined in the EU-funded project WELCOME (Wearable Sensing and Smart Cloud Computing for Integrated Care to COPD Patients with Comorbidities) [1], for the integrated management of COPD and comorbidities.
\end{abstract}

Keywords; COPD, multimorbidity, integrated care, cloud computing

\section{INTRODUCTION}

According to WHO [2], "Integrated care is a concept bringing together inputs, delivery, management and organization of services related to diagnosis, treatment, care, rehabilitation and health promotion. Integration is a means to improve services in relation to access, quality, user satisfaction and efficiency". This implies integration across disciplines and aspects of health to address patient status as a whole, especially in the elder multimorbid chronic patients, and integration along services provided from home to hospital.

The focus in this work is Chronic Obstructive Pulmonary Disease (COPD) and its common comorbidities (heart failure, diabetes and depression), as studied in the project WELCOME. This is due to the prevalence of these multimorbid chronic conditions among the increasing elder population, and therefore the expected impact of such integrated care approaches, as well as due to its paradigmatic nature with a series of intertwined physiological conditions that need management. The challenge is to employ as much as possible ubiquitous monitoring of patient's condition, and present the health professionals with valuable but not overwhelming information, as regards the overall patient's status and trends. This includes information on cardiac and lung function, patient's mobility and depression status, as well as correct use of medication. These pieces of information would then be combined in order to detect deteriorations, and additionally to trace the causes of deterioration, which is extremely important for the timely and efficient treatment and management of multimorbid patients. For example, a combination of deteriorating / stable signs and signals should suggest the probable causative factors and the required course of action. 
A number of enabling ICT technologies need to be put inplace, in order to address these needs for acquisition, transmission, storage, analysis, decision support, user interaction. A core component will be the sensors deployed on wearable technologies (vest) for recording and streaming biodata to the mobile patient hub (e.g. a tablet), along with a pick mix of standard Bluetooth-enabled sensors for periodic measurements (e.g. weight) also connected to the patient-hub. The WELCOME smart cloud platform is the heart of the system where all the medical records and the monitoring data are managed and processed. The WELCOME cloud platform consists of several modules (storage server, feature extraction module, decision support system and external applications connector) and the orchestrator. This paper gives an overview of the cloud-based processing of the data streams towards detecting changes in patient status and supporting decisions within an integrated care context.

\section{METHODS}

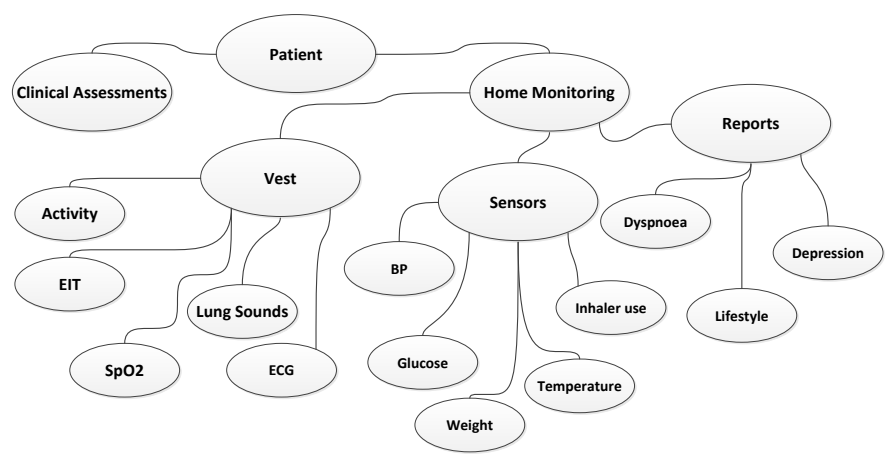

Figure 1. Overview of the Home Monitored data in WELCOME

An overview of the parameters monitored is depicted on Figure 1, focusing on the patient monitored data, rather than data acquired during usual clinical procedures. These homebased monitoring data consist of streaming biosignals, single bioparameters acquired per day, and patient-reported answers.

\section{A. User Input and Example Scenarios}

A series of scenarios have been elaborated by clinical partners to illustrate the features that need to be calculated, the types of rules that need to fire, and the foreseen clinical benefit of the system, in handling COPD and comorbidities.

Example scenario 1. For patient $\mathrm{X}$, in the last 5-7 days the data from the vest have changed. Mean heart rate (HR) is 85$100 /$ min especially late at night, and mean respiratory rate (RR) is $17-19 / \mathrm{min}$. ECG shows sinus tachycardia. $\mathrm{SpO}_{2}$ is $97 \%$. There is no change in lung sounds. Temperature is normal. Actigraphy shows no reduction of activity. Electrical Impedance tomography (EIT) shows no changes. Patient is advised for continuous monitoring through the vest. In the next 2 days, the vital signs are the same with no changes in lung sounds, EIT, or SpO2. Sinus tachycardia remains especially during late at night without any other ECG changes, with RR $18-20 / \mathrm{min}$ most time of the day. Dyspnoea (MRC breathlessness scale) increases to 1. Actigraphy shows reduction of activity. The system informs the informal carer, the psychiatrist and the pulmonologist about the changes. The patient is advised to visit the psychiatrist.

Example Scenario 2. Patient $\mathrm{Y}$ is diagnosed with COPD/ CHF stage III according to GOLD/ NYHA. She wears the Vest $6 \mathrm{~h}$ during day and $8 \mathrm{~h}$ at sleep. On Day 1 , vital signs gradually deteriorate during the last 8 hours: $\mathrm{RR}=18$ to $21 / \mathrm{min}, \mathrm{HR}=85$ to $101 / \mathrm{min}, \quad \mathrm{SpO}_{2}=95 \%$ (baseline $=96 \%$ ), Temperature $=$ $37,3^{\circ} \mathrm{C}$. ECG shows sinus tachycardia. Lung sounds show a slight prolongation of exhalation time. Cough events are detected. EIT shows no difference from baseline. Patient is advised to take rescue inhaler therapy (extra puffs) and to continue wearing the monitoring vest. Patient is prompted for input on dyspnea/sputum description but patient ignores it. Informal carer is informed of potential onset of acute exacerbation. On Day 2, vital signs deteriorate, reaching on average: $\quad \mathrm{RR}=25 / \mathrm{min}, \quad \mathrm{HR}=103 / \mathrm{min}, \quad \mathrm{SpO}_{2}=90 \%$ (baseline $=96 \%$ ), Temperature $=37,8^{\circ} \mathrm{C}, \mathrm{BP}=155 / 94 \mathrm{mmHg}$. ECG shows sinus tachycardia. Chest sounds show slight prolongation of exhalation time, cough, and crackles in the basal parts of lungs unilaterally. EIT shows prolonged expiration and increased ventilation heterogeneity (possible pleural effusion). Patient Y reports increased dyspnea, body weight $=2 \mathrm{Kg}$ more than 2 days ago, increased and yellow sputum production. System informs pulmonologist and cardiologist about the progress of the symptoms. MDs acknowledge a new acute exacerbation and decide to activate the prompt for visit to the hospital, and via the system, prompt patient and informal carer to increase diuretics dosage.

TABLE I. CHARACTERISTIC SIGNALS AND FEATURES

\begin{tabular}{|c|c|c|}
\hline Signal/Source & Feature & Usage \\
\hline Lung Sounds & $\begin{array}{l}\text { Daily Number of cough } \\
\text { events, crackle events, } \\
\text { wheezing events }\end{array}$ & COPD \\
\hline ECG & $\begin{array}{l}\text { Heart Rate, AF events, } \\
\text { tachy/bradycardia }\end{array}$ & Arrhythmias \\
\hline Actigraphy & $\begin{array}{l}\text { Daily nonsleeping/sitting } \\
\text { activity }\end{array}$ & $\begin{array}{l}\text { Depression, } \\
\text { COPD or HF } \\
\text { worsening }\end{array}$ \\
\hline EIT & $\begin{array}{l}\text { Regional airflow metrics } \\
\text { (FEV1/FVC) }\end{array}$ & $\begin{array}{c}\text { COPD } \\
\text { exacerbation }\end{array}$ \\
\hline Inhaler usage & Daily use, erroneous use & COPD \\
\hline Depression scale & Periodically & Depression \\
\hline Dyspnoea scale & $\begin{array}{l}\text { Periodically and upon } \\
\text { demand }\end{array}$ & COPD \\
\hline $\begin{array}{l}\text { Lifestyle pick and } \\
\text { mix questions }\end{array}$ & Periodically & $\begin{array}{c}\text { Diabetes and } \\
\text { overall lifestyle }\end{array}$ \\
\hline $\begin{array}{l}\text { Weight, Glucose, } \\
\text { BP, Temperature }\end{array}$ & Daily value & $\begin{array}{c}\text { Diabetes, } \\
\text { Inflammation, HF } \\
\text { worsening }\end{array}$ \\
\hline
\end{tabular}

\section{B. System Overview: Flow of Data and Features}

The data depicted in Figure 1 are initially stored on the cloud storage server, with the procedure depicted in Fig $2 \mathrm{a}$. These are later employed in a feature extraction process, in order to detect clinically meaningful parameters, with respect to the clinical scenarios, as described above. Fig $2 \mathrm{~b}$ depicts the scenario where the analysis of the acquired biosignals by the Feature Extraction Engine and the results are stored back in the storage server in order to be available to the system's users, 
takes place. An overview of characteristic features and their context is presented in Table 1, while more details on the features are presented in the next subsections. It is worth noting that feature analysis includes calculations at different scales, i.e. single events (detection of cough), features in a timeframe (HR), events per day, mean values per day, trends, and differences from absolute or previous days baseline.

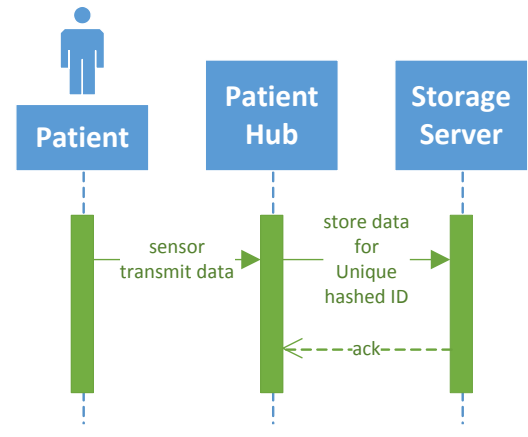

(a)

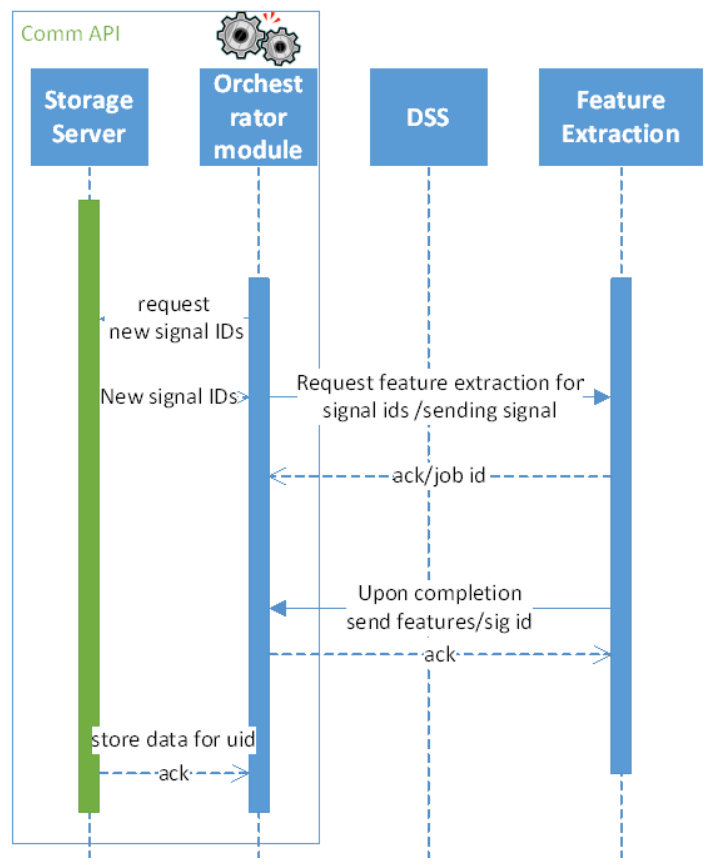

(b)

Figure 2. (a) The transmission of data from the patient based sensors to the storage server.(Actor=Patient), (b) The bio signal processing and feature extraction procedure.(Actor=Orchestrator module)

Then, the generated features are employed in the Decision Support System (DSS) module, in an information flow analogous to the one described for the feature extraction engine. The firing of rules will require the coordinated use of the feature extraction engine and the DSS, and the orchestrator module plays this role. Of note, the communication among the components of the WELCOME system is performed via RESTful Web services over HTTPS (and VPN).

\section{Biosignals and Feature Extraction}

Lung Sounds. The analysis of respiratory sounds and respiratory manoeuvres, such as cough, is a valuable diagnostic tool for the detection and follow-up of respiratory diseases such as chronic obstructive pulmonary disease (COPD). Respiratory sounds can be classified as breath sounds, abnormal breath sounds and adventitious sounds [3]. Adventitious sounds refer to additional respiratory sounds superimposed on breath sounds. These sounds include wheezes (continuous sounds), stridors, squawks and crackles (discontinuous sounds).

In this work, we describe our preliminary attempts towards automatic crackle and cough detection. In both tasks we propose to attain three main goals: i) to create a sizeable and meaningful dataset for algorithm evaluation; ii) to identify and propose robust audio features for each of the problems; iii) and to take advantage of a multi-sensor setup, where blind source separation techniques will be explored to separate the multiple information sources provided in the auscultation signals. In fact, sounds such as crackles are easily masked by other respiratory and background sounds (e.g. deep breaths). Thus, we believe this separation process will allow for an accurate detection and characterization of different respiratory sounds.

Regarding crackles, these are short explosive sounds that seem to result from an abrupt opening or closing of the airways [4]. Crackles can usually be classified based on their total duration (2CD) as fine (<10 ms) or coarse (>10 ms) [5]. So far, we employed two datasets available on-line [6] (first channel of the repository "Crackle (a)" and the repository "Crackle (c)") of respiratory sounds containing crackles. Features extracted from the Teager energy operator [7], the wavelet packed stationary transform - no stationary transform (WPST-NST) [8], complexity measurement by the fractal dimension [9], the sum of Intrinsic Mode Functions [10], Information Entropy [11] and the Generalized Autoregressive Conditional Heteroskedasticity (GARCH) Process [12], were evaluated, namely, statistical features such as the maximum, minimum and quartiles from each of the previous processes.

As for cough, this is a respiratory reflex characterized by sudden expulsion of air at a high velocity accompanied by a transient sound of varying pitch and intensity [5]. Cough is a common and important symptom in many respiratory diseases. As before, we evaluated a number of audio features, namely, Mel Frequency Cepstral Coefficients (MFCC), sound pitch, spectral rolloff and spectral inharmonicity [13].

Electrical Impedance Tomography. One of the most innovative and novel features implemented in WELCOME is monitoring the patient's regional lung ventilation through a medical imaging modality, known as electrical impedance tomography (EIT). EIT is a non-invasive, radiation-free medical imaging technique that will become wireless and wearable through the WELCOME project.

In lung EIT, a set of electrodes is placed around the patient's thorax and used for injecting electrical currents and measuring the resulting potentials through well-defined stimulation patterns $[14,15]$. These potentials are used for the computation of images showing the distribution of electrical resistivity changes in the studied chest cross-section. These images constitute a regularized inverse solution of the generalized Laplace equation [16], a highly nonlinear ill-posed problem [17]. 


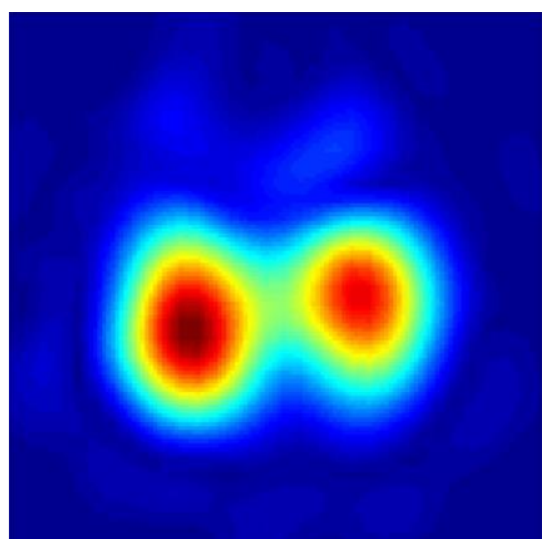

Figure 3. Functional EIT image showing the distribution of tidal ventilation in a seated, spontaneously breathing man.

Assessment of regional lung ventilation is one of the most promising applications of EIT because large volumes of air are moved in and out of the lungs during breathing, resulting in measurable changes in lung tissue resistivity. An example of a functional EIT image showing the distribution of ventilation in a healthy subject is depicted in Figure 3. Well ventilated lung regions exhibit high resistivity variation (red) and, inversely, regions with absent ventilation are depicted as areas of low variation (blue). In spite of the low spatial resolution of EIT images, several clinical studies have shown that useful quantitative information about, e.g., lung ventilation or respiratory system mechanics, can be extracted $[18,19]$. This is justified, in part, by the high temporal resolution of the current EIT systems (ranging from 13 to 50 frames per second).

So far, lung EIT has mainly been used as a tool for the determination of the least injurious mechanical ventilator settings in intensive care units [20]. A recent clinical study on COPD patients has shown that EIT can detect the effects of regional airway obstruction during pulmonary function testing [21]. In WELCOME, in addition to spontaneous tidal breathing monitoring, standard ventilation manoeuvres performed are foreseen. Early results have shown that regional ratios of forced expiratory volume in $1 \mathrm{~s}\left(\mathrm{FEV}_{1}\right)$ and forced vital capacity (FVC) can be computed using the acquired EIT image sequences, while classical spirometry gives only one global $\mathrm{FEV}_{1} / \mathrm{FVC}$ value. New indices characterizing the spatial lung function heterogeneity in COPD may be further developed. As an example, we may consider the frequency distributions of pixel $\mathrm{FEV}_{1} / \mathrm{FVC}$ ratios which successfully depict the heterogeneity of lung disease in COPD compared with healthy subjects [21]. Active research aims to establish and standardize these new indices in order to be used as indicators of COPD progression and, also, as detectors of early stages of exacerbations. To this direction, EIT findings can be combined with other features (e.g. lung sounds and heart rate) for the design of more robust exacerbation detection rules.

Inhaler Compliance Analysis: A monitoring device has been designed to record the audio of inhaler use. The audio recordings can be employed to monitor when the inhaler was used as well as evidence of flow rates achieved by the user. The INCA device is attached to a Diskus inhaler. It begins recording as soon as the inhaler is opened and stops recording when the inhaler is closed and saves the recording on a local memory. The resultant audio recording is saved with the time and date of the inhaler actuation. In addition, the audio can be processed to identify any technique errors that can occur and the flow rate achieved during inhalation. Common technique errors include poor inspiration effort, exhaling into the mouth piece after loading the drug and multiple respirations and these can all be identified from processing the audio signal. Attempts to use the inhaler are saved over the course of a month when the device is removed from the inhaler, audio recordings downloaded and processed [22]. The output of the processing is the traffic light graph in Figure 4, where each inhaler event is marked with a green or red dot, taken correctly or incorrectly respectively. This information is used as feedback for the users to educate them on potential technique errors and improving the temporal adherence to their medication regime.

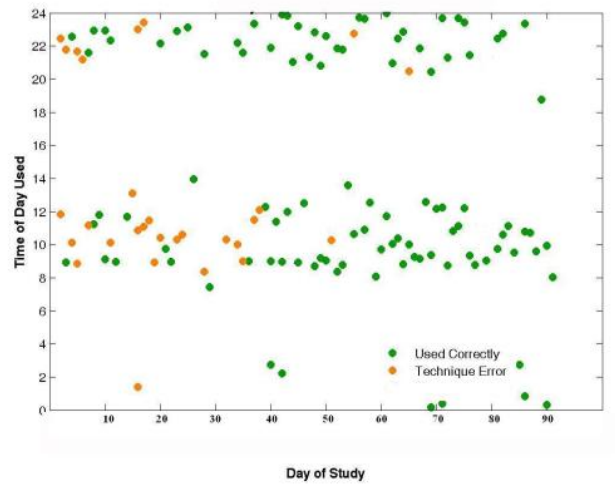

Figure 4. Inhaler events (here two per day), colored coded according to the corrected or erronous inhaler use.

\section{RESUlTS AND Discussion}

The current work aims at presenting the basic rationale and research methods for cloud based multiparametric analysis and decision support in the management of complex multimorbid conditions. While the cloud feature extraction and the DSS system development is ongoing work, preliminary results on the WELCOME cloud computing development include:

- Formulation of the concepts, constraints, and high level rules based on clinical input and clinical scenarios. An ontology of these concepts is ongoing work, towards semantic reasoning based on the high level calculated features at the required time scales. Specifically, this ontology will be used to define the data model, to validate the data against specific semantically enriched rules and enhance the DSS module with semantic reasoning capabilities.

- Preliminary results for lung sound analysis. As regards Crackles, the Matthews correlation coefficient (MCC), measured after classifying the data using the Logistic Regression classifier, was used to evaluate the capacity of the different features (or combination of features) to detect segments with coarse crackles. Preliminary results show 0.8 correlation and suggest the third quartile of information entropy as the most descriptive feature. For cough detection, using a simple Nearest 
Neighbor classifier, with $\mathrm{k}=11$ neighbors, we attained $97.4 \%$ recall and $85.4 \%$ precision scores.

- Data Collection for lung algorithm validation. As for crackles data acquisition, we are presently collecting and annotating hospital data, acquired from the Centro Hospitalar e Universitário de Coimbra. This is work in progress. In parallel, data are collected for the cough detection validation. So far, 14 minutes of audio data from 16 volunteers (10 healthy, 5 with a cold and 1 with bronchitis) were collected and annotated, amounting for 112 cough events.

- EIT image reconstruction with existing datasets and calculation of basic volume features.

- Effort to incorporate the standalone inhaler analysis in the integrated framework.

A series of challenges pertain in creating a semantic model of all data features, constraints and rules [23], in completing the analysis and decision support tasks and integrating the algorithms in the cloud framework. Among the future steps will be the wider validation of algorithms, the robustness and performance analysis, as well as the implementation of the full DSS based on the calculated features.

\section{ACKNOWLEDGMENT}

The whole WELCOME consortium is acknowledged for their inputs and support.

\section{REFERENCES}

[1] http://www.welcome-project.eu/home.aspx

[2] O Gröne and M Garcia-Barbero, "Trends in Integrated Care Reflections on Conceptual Issues", World Health Organization, Copenhagen, 2002, EUR/02/5037864.

[3] A. R. A. Sovijärvi, L. P. Malmberg, G. Charbonneau, J. Vanderschoot, F. Dalmasso, C. Sacco, M. Rossi, and J. E. Earis, "Characteristics of breath sounds and adventitious respiratory sounds," Eur Respir Rev, vol 10(77), pp. 591-596, 2000

[4] P. Piirilä and A. R. A. Sovijärvi, "Crackles: recording, analysis and clinical significance,” Eur. Respir. J., vol. 8(12), pp. 2139-48, 1995.

[5] A. R. A. Sovijärvi, F. Dalmasso, J. Vanderschoot, L. P. Malmberg, and G. Righini, "Definition of terms for applications of respiratory sounds," Eur. Respir. Rev., vol. 10(77), pp. , 597-610,2000.

[6] "R.A.L.E. Repository" [Online]. Available: http://www.rale.ca. [Accessed: 10-Mar-2014].

[7] E. Kvedalen, "Signal processing using the Teager energy operator and other nonlinear operators," Master Thesis, Dep. Informatics, Univ. Oslo, Norway, 2003.
[8] M. Bahoura and X. Lu, "Separation of crackles from vesicular sounds using wavelets packet transform," in 2006 Proc. Int. Conf. on Acoustics, Speech, and Signal. vol.2, pp.II,II, 14-19 May 2006

[9] M. J. Katz, "Fractals and the analysis of waveforms.," Comput. Biol. Med., vol. 18(3), pp. 145-56, Jan. 1988.

[10] N. E. Huang, Z. Shen, S. R. Long, M C. Wu, H. H. Shih, Q. Zheng, N. Yen, C. C Tung and H. H. Liu, "The empirical mode decomposition and the Hilbert spectrum for non-linear and non-stationary time series analysis," Proc. Roy. Soc. London, vol. 454,. pp. 903-95, Mar 1998.

[11] R. C. Gonzalez, R. E. Woods, and S. L. Eddins, Digital Image Processing Using Matlab. Gatesmark Publishing, 2004.

[12] "Time series analysis." [Online]. Available: http://www.math.kth.se/ matstat/gru/sf2943/ts.pdf. [Accessed: 07-Mar-2014].

[13] O. Lartillot and P. Toiviainen, "A Matlab Toolbox for Musical Feature Extraction from Audio", Proc. 10th Int. Conf. on Digital Audio Effects, pp. DAFX1-8, 2007.

[14] D.C. Barber, B.H. Brown, “Applied Potential Tomography”, Journal of Physics E: Scientific Instruments, vol 17(9), pp. 723-733, 1984.

[15] M. Bodenstein, M. David, and K. Markstaller, "Principles of electrical impedance tomography and its clinical application", Critical Care Medicine, vol 37(2), p. 713-24, 2009.

[16] A. Adler, JH Arnold, R Bayford, A Borsic, B Brown et al, "GREIT: a unified approach to 2D linear EIT reconstruction of lung images", Physiol. Meas, vol 30(6),pp. S35-55, 2009.

[17] A. Adler, MB Amato, JH Arnold, R Bayford, M Bodenstein et al, "Whither lung EIT: Where are we, where do we want to go and what do we need to get there?", Physiol. Meas, vol 33(5), pp. 679-94, 2012.

[18] I. Frerichs, "Electrical impedance tomography (EIT) in applications related to lung and ventilation: a review of experimental and clinical activities", Physiol. Meas. vol 21(2), pp. R1-21, 2000.

[19] I. Frerichs, T. Becher, and N. Weiler, "Electrical impedance tomography imaging of the cardiopulmonary system", Curr Opin Crit CareVol 20, pp. 323-332, 2014

[20] C Gomez-Laberge, J.H. Arnold, and G.K. Wolf, "A Unified Approach for EIT Imaging of Regional Overdistension and Atelectasis in Acute Lung Injury”, IEEE Trans. On Medical Imaging, vol 31(3) pp. 834-42, 2012 .

[21] B. Vogt, S. Pulletz, G. Elke, Z. Zhao, P. Zabel, N. Weiler, and I. Frerichs, "Spatial and temporal heterogeneity of regional lung ventilation determined by electrical impedance tomography during pulmonary function testing”, J Appl Physiol, vol 113(7), pp.1154-61, 2012.

[22] S D'Arcy, E MacHale, J Seheult, MS Holmes, C Hughes, et al, “A Method to Assess Adherence in Inhaler Use through Analysis of Acoustic Recordings of Inhaler Events“, PLoS ONE vol 9(6), pp. e98701, 2014.

[23] SR Abidi, “A Conceptual Framework for Ontology Based Automating and Merging of Clinical Pathways of Comorbidities", Knowledge Management for Health Care Procedures, Lecture Notes in Computer Science, Springer Berlin Heidelberg, Vol 5626, pp. 55-66, 2009 